\title{
Novas ocorrências de liquens corticícolas crostosos e microfoliosos em vegetação de Caatinga no semi-árido de Alagoas
}

Aline Anjos de Menezes ${ }^{1}$, Amanda Barreto Xavier Leite ${ }^{1}$, Amanda Yumi Otsuka ${ }^{1}$, Luciana Santos de Jesus ${ }^{1}$ e Marcela Eugenia da Silva Cáceres ${ }^{2}$

\author{
Recebido em 13/02/2011. Aceito em 1/10/2011
}

\begin{abstract}
RESUMO
(Novas ocorrências de liquens corticícolas crostosos e microfoliosos em vegetação de Caatinga no semi-árido de Alagoas). São apresentados 25 novos registros para o Estado (toponímia) de Alagoas, sendo uma espécie novo registro para a região Nordeste e quatro outras novas ocorrências para o Brasil. Uma lista com todas as espécies atualmente conhecidas para o semi-árido de Alagoas é apresentada. As amostras foram coletadas na região do Município de São José da Tapera, estado de Alagoas, onde ocorre uma rica vegetação de Caatinga arbóreo-arbustiva.az de regenerar e recrutar em ambientes sombreados, mas o crescimento é limitado pelas condições de pouca luminosidade.
\end{abstract}

Palavras-chave: liquens, Ascomycota, Caatinga, diversidade, novos registros

\begin{abstract}
(New records of crustose and microfoliose corticicolous lichens in Caatinga vegetation of the semi-arid region in Alagoas). A total of 25 species are here reported for the first time for Alagoas State, with one new record for the Northeastern Region of Brazil and four new records for Brazil. A list of all known species for the semi-arid region in Alagoas is presented. Sampling took place near the municipality of São José da Tapera, in the semi-arid region of Alagoas, where a very rich Caatinga vegetation of trees and shrubs occurs.
\end{abstract}

Key words: lichens, lichenized Ascomycota, Caatinga, diversity, new records

\section{Introdução}

Os liquens fazem parte de um grupo extremamente diversificado de fungos, encontrados na natureza simbioticamente associados a algas e/ou cianobactérias (Webster \& Weber 2007). O processo de associação é denominado liquenização e ocorre em mais de $20 \%$ de todas as espécies de fungos conhecidas, representando aproximadamente $46 \%$ dos fungos do Filo Ascomycota (Nash 2008). Os fungos liquenizados exercem diferentes funções nos ecossistemas, assim como são importantes algumas de suas aplicações pelo homem, por exemplo, o uso destes organismos como biondicadores de poluição e perturbação antrópica.

Liquens podem ocorrer em vários substratos e ambientes. Podem se fixar em troncos e suas ramificações, no solo, sobre rochas ou folhas, e às vezes até sobre substratos menos convencionais como o plástico (Lücking 1998). Os liquens são encontrados em ambientes luminosos (espécies heliófitas) ou sombrios (espécies umbrófitas), assim como são capazes de colonizar ambientes extremos em umidade e temperatura (Lemos et al. 2007). Variam em sua complexidade de formas de crescimento, separados basicamente em liquens crostosos, foliosos e fruticosos, com algumas variações destes tipos básicos como os talos esquamulosos e filamentosos (Büdel \& Scheidegger 2008)

Atualmente, estudos sobre a diversidade e ecologia de liquens no Brasil são escassos, principalmente fora das regiões Sul e Sudeste (Cáceres 2007). Há hoje pouquíssimos profissionais especialistas em taxonomia de liquens no país, ocorrendo uma distribuição desigual na produção científica de acordo com os grupos estudados e regiões visitadas. Dentre os trabalhos realizados no Brasil, destacam-se estudos sobre a diversidade de fungos liquenizados foliosos na região sudeste (Marcelli 1997), incluindo áreas de manguezal no estado de São Paulo (Benatii \& Marcelli 2007), e estudos recentes sobre as famílias Parmeliaceae e Physciaceae no mesmo estado (Benatti \& Marcelli 2008, Jungbluth \& Marcelli 2008, 2011a, b, Marcelli et al. 2011). Na região Sul, tem sido estudado também a diversidade de liquens foliosos (Eliasaro 2008, Spielmann 2006, Spielmann

\footnotetext{
Universidade Federal de Sergipe, Departamento de Biociências, Itabaiana, SE, Brasil

2 Autor para correspondência: mscaceres@hotmail.com
} 
\& Marcelli 2008b) e crostosos (Käffer \& Mazzitelli 2005, Käffer et al. 2010), além de trabalhos importantes sobre a Família Graphidaceae (Dal-Forno 2009, Dal-Forno \& Eliasaro 2010a, b).

Dentre os estudos taxonômicos e de diversidade de liquens no Norte e Nordeste brasileiro, destacam-se trabalhos e projetos recentes relacionados com a taxonomia de liquens foliícolas e corticícolas crostosos da Mata Atlântica, Amazônia Oriental e Semi-árido (Cáceres 2007, Cáceres et al. 2000, 2007, 2008a, b; Lücking \& Cáceres 2004).

O presente trabalho tem como objetivo apresentar as novas ocorrências de liquens corticícolas crostosos para a região semi-árida do estado de Alagoas, para o Nordeste e para o Brasil, como resultado da realização de projetos de pesquisa em andamento sobre o estudo da diversidade destes organismos na região, com ênfase no estudo da diversidade liquênica em áreas de Caatinga.

\section{Materiais e métodos}

Coletas foram realizadas em uma fazenda na região do município de São José da Tapera, localizada no Estado de Alagoas. A área municipal ocupa $519,64 \mathrm{~km}^{2}$, estando inserida na mesorregião do Sertão Alagoano e na microrregião de Santana do Ipanema. Uma parte de sua área (cerca de $40 \%$ ) está inserida na unidade geoambiental da Depressão Sertaneja, que representa a paisagem típica do semi-árido nordestino. A vegetação é basicamente composta por Caatinga Hiperxerófila com trechos de Floresta Caducifólia. O clima é do tipo Tropical Semi-Árido, com chuvas de verão, o qual se inicia em novembro com término em abril, apresentando uma precipitação média anual é de $431,8 \mathrm{~mm}$ (Mascarenhas et al. 2005).

Os liquens foram coletados em ramos e troncos de árvores e de arbustos aleatórios em área de vegetação típica de Caatinga arbórea, em uma propriedade localizada no Município de São José da Tapera $\left(9^{\circ} 33^{\prime} 28,8^{\prime \prime}\right.$ S e $\left.37^{\circ} 22^{\prime} 51,6^{\prime \prime} \mathrm{W}\right)$, no estado de Alagoas. Esta localidade faz fronteira com os municípios de Senador Rui Palmeira Carneiros, Pão de Açúcar, Monteirópolis, Olho d' Água das Flores e Piranhas. Foram coletadas 171 amostras durante uma expedição em Junho de 2008.

As amostras foram identificadas no Laboratório de Micologia, Departamento de Biociências da Universidade Federal de Sergipe, Campus Professor Alberto Carvalho, em Itabaiana, Sergipe. A identificação foi feita através de características observadas no talo liquênico de todas as amostras, como as seguintes: hábito, formato, esporos (coloração e tamanho), reações ao Iodo (I) e/ou Hidróxido de Potássio $(\mathrm{KOH})$. As devidas observações foram feitas em lupa e através de cortes transversais das amostras. Posteriormente as lâminas foram observadas em microscópio óptico e tratadas com I e/ou KOH. Todos esses procedimentos serviram como respaldo para a identificação das espécies de acordo com a seguinte bibliografia: Cáceres (2007), Lücking et al. (2009), Lücking \& Rivas-Plata (2008), Marbach (2000), entre outros.

\section{Resultados e discussão}

Um total de 171 amostras de liquens corticícolas crostosos foram analisadas, das quais 51 espécies foram identificadas (Tabela 1). Com base nos escassos trabalhos publicados sobre liquens no estado de Alagoas, que foram realizados somente em áreas de Mata Atlântica (Cáceres 2007, Cáceres et al. 2007, 2008a, b, Pereira et al. 2006), um total de 25 táxons representam novos registros para o estado de Alagoas (Tabela 1), sendo uma espécie registrada pela primeira vez para a região Nordeste e quatro consideradas novas ocorrências para o Brasil (Cáceres 2007, Lücking et al. 2009, Frisch 2006).

Com relação às espécies crostosas citadas aqui pela primeira vez para o estado de Alagoas, a maioria já havia sido previamente citada para outros estados do Nordeste (Cáceres 2007). Baculifera pseudomicromera Marbach, B. endochlora (J. Steiner) Marbach, Cratiria obscurior (Stirton) Marbach \& Kalb, Hafellia curatellae (Malme) Marbach, $H$. demutans (Stirton) Pußwald, H. desertica Marbach, Lecanora caesiorubella Ach., Pertusaria tetrathalamia (Fée) Nyl., Polymeridium proponens (Nyl.) R. C. Harris, Pseudopyrenula subnudata Müll. Arg., Vainionora flavovirens (Fée) Kalb foram citadas anteriormente para o estado de Pernambuco (Cáceres 2007). Para Sergipe, Cáceres (2007) já havia citado Glyphis substriatula (Nyl.) Staiger, Lecanora hypocrocina Nyl. e Stigmatochroma gerontoides (Stirt.) Marbach. Já Haematomma personii (Fée) A. Massal., Helminthocarpon leprevostii Fée, Pertusaria flavens Nyl. e Ramboldia haematites (Fée) Kalb haviam sido previamente registradas para os estados de Pernambuco e Sergipe (Cáceres 2007). Coenogonium moniliforme Tuck., que apresenta talo de aparência crustosa (Rivas Plata et al. 2006), porém finamente filamentoso, já havia sido citado anteriormente para Pernambuco (Cáceres 2007) e foi encontrado também em Sergipe (Cáceres, dados não publicados), na região Nordeste.

As espécies de liquens microfoliosos consideradas como primeiro registro para o estado de Alagoas são Heterodermia dissecta (Kurok.) D. D. Awasthi, Pyxine berteriana (Fée) Imshaug, Physcia sorediosa (Vain.) Lynge e Dirinaria confluens (Fr.) D. D. Awashti. Entende-se que estas espécies apresentem uma distribuição ampla, como no caso de $D$. confluens (Awasthi 1975), porém não haviam até o momento sido formalmente citadas para o estado, principalmente devido ao pioneirismo deste trabalho no semi-árido alagoano, ambiente onde predomina a família Physciaceae (Cáceres et al. 2008a).

Para o Nordeste, o novo registro foi Heterodermia albicans (Pers.) Swinscow \& Krog, espécie também microfoliosa, previamente citada para o Sul e Sudeste do país (Eliasaro et al. 2009; Martins et al. 2008; Spielmann 2006).

As espécies Graphis seminuda Müll. Arg, G. handelii Zahlbr., G. glauconigra Vain. e Leucodecton occultum (Eschw.) A. Frisch são novos registros para o Brasil. Graphis 
Tabela 1. Lista de espécies de liquens crostosos registrados na região semi-árida do estado de Alagoas. $\mathrm{AL}=$ nova ocorrência para Alagoas; $\mathrm{BR}=$ nova ocorrência para o Brasil; NE = nova ocorrência para o nordeste.

\begin{tabular}{|c|c|c|c|}
\hline Família/Espécies & Nova Ocorrência & Família/Espécies & Nova Ocorrência \\
\hline Arthoniaceae & & Baculifera pseudomicromera Marbach & $\mathrm{AL}$ \\
\hline Arthonia aff. complanata Fée & & Baculifera xylophila (Malme) Marbach & $\mathrm{AL}$ \\
\hline Arthonia sp. & & Cratiria obscurior (Stirton) Marbach \& Kalb & $\mathrm{AL}$ \\
\hline Coniocarpon cinnabarinum DC. & & Dirinaria confluens (Fr.) D. D. Awasthi & $\mathrm{AL}$ \\
\hline \multirow[t]{2}{*}{ Helminthocarpon leprevostii Fée } & $\mathrm{AL}$ & Hafellia bahiana (Malme) Sheard & \\
\hline & & Hafellia curatellae (Malme) Marbach & $\mathrm{AL}$ \\
\hline Chrysothrichaceae & & Hafellia demutans (Stirton) Pußwald & $\mathrm{AL}$ \\
\hline Chrysothrix chlorina (Ach.) J. R. Laundon & $\mathrm{AL}$ & Hafellia desertica Marbach & $\mathrm{AL}$ \\
\hline \multirow[t]{2}{*}{ Chrysothrix sp. } & & Heterodermia albicans (Pers.) Swinscow \& Krog & $\mathrm{NE}$ \\
\hline & & Heterodermia dissecta (Kurok.) D. D. Awasthi & $\mathrm{AL}$ \\
\hline Roccelaceae & & Physcia sorediosa (Vain.) Lynge & $\mathrm{AL}$ \\
\hline \multirow[t]{2}{*}{ Opegrapha cf. arengae Vain. } & & Pyxine berteriana (Fée) Imshaug & $\mathrm{AL}$ \\
\hline & & Rinodina sp. & \\
\hline Trypetheliaceae & & Stigmatochroma gerontoides (Stirton) Marbach & $\mathrm{AL}$ \\
\hline Polymeridium proponens (Nyl.) R. C. Harris & $\mathrm{AL}$ & Graphidaceae & \\
\hline Pseudopyrenula subnudata Müll. Arg. & $\mathrm{AL}$ & Glyphis cicatricosa Ach. & $\mathrm{AL}$ \\
\hline Trypethelium ocroleucum (Eschw.) Nyl. & & Glyphis scyphulifera (Ach.) Staiger & BR \\
\hline \multirow[t]{2}{*}{ Trypethelium eluteriae Spreng. } & & Glyphis substriatula (Nyl.). Staiger & BR \\
\hline & & Graphis seminuda Müll. Arg & $\mathrm{BR}$ \\
\hline Pyrenulaceae & & Graphis handelii Zahlbr. & \\
\hline \multirow[t]{2}{*}{ Pyrenula anomala (Ach.) Vain. } & $\mathrm{AL}$ & Graphis glauconigra Vain. & \\
\hline & & Graphis pavoniana Fée & \\
\hline Lecanoraceae & & Graphis palmyrensis Zahlbr. & $\mathrm{AL}$ \\
\hline Haematomma personii (Fée) A. Massal. & $\mathrm{AL}$ & Phaeographis sp. & \\
\hline Lecanora sp. & & Coenogonium moniliforme Tuck. & \\
\hline \multicolumn{4}{|l|}{ Lecanora achroa Nyl. } \\
\hline Lecanora caesiorubella Ach. & $\mathrm{AL}$ & Pertusariaceae & \\
\hline Lecanora hypocrocina Nyl. & $\mathrm{AL}$ & Pertusaria dehiscens Müll. Arg. & $\mathrm{AL}$ \\
\hline Ramboldia haematites Körb. & $\mathrm{AL}$ & Pertusaria flavens Nyl. & $\mathrm{AL}$ \\
\hline Vainionora flavoriens (Fée) Kalb. & $\mathrm{AL}$ & Pertusaria tetrathalamia (Fée) Nyl. & $\mathrm{AL}$ \\
\hline Physciaceae & & Thelotremataceae & \\
\hline Baculifera entochlora (J. Steiner) Marbach & $\mathrm{AL}$ & Leucodecton occultum (Eschw.) A. Frisch. & BR \\
\hline
\end{tabular}

seminuda foi descrita para a Austrália como G. catherinae A. W. Archer (Archer \& Elix 2007) e posteriormente registrada para a Costa Rica, já com a nova combinação (Lücking et al. 2008). Graphis handelii havia sido citada para o Paraguay, China e Índia antes deste trabalho (Lücking et al. 2009) e G. glauconigra era conhecida antes apenas na China, Índia e Filipinas (Lücking et al. 2009). Já Leucodecton occultum havia sido descrita para a África (Frisch 2006), e depois só registrada na Austrália e Galápagos (GBIF 2010).

Dos 22 gêneros representados no trabalho, 12 são novos registros para o Estado de Alagoas: Baculifera Marbach \& Kalb, Cratiria Marbach, Helmintocarpon Fée, Heterodermia Trevis., Pertusaria DC., Leucodecton A. Massal., Physcia (Schreb.) Michx., Polymeridium (Müll. Arg.) R.C. Harris, Pseudopyrenula Müll. Arg., Pyxine Fr., Stigmatochroma
Marbach e Vainionora Kalb. Os gêneros mais diversos são Baculifera, Graphis Adans., Hafellia Kalb, H. Mayrhofer \& Scheid. e Pertusaria DC., todos com três espécies cada.

Os 25 novos registros de liquens para Alagoas estão distribuídos em 10 famílias sendo que a família que possui o maior número de espécies é Physciaceae, com 12 espécies (38,7\%), seguida de Lecanoraceae, com cinco espécies $(16,12 \%)$ e Graphidaceae, com quatro (12,9\%).

$\mathrm{O}$ trabalho constitui o primeiro registro de fungos liquenizados na região semi-árida do Estado de Alagoas, com vegetação de Caatinga. É uma importante contribuição ao conhecimento da micota liquenizada do estado como um todo, ainda praticamente inexplorada, e do Brasil, com o acréscimo de quatro novos registros de espécies de liquens para o país e uma para a região Nordeste. 
Outras áreas ainda precisam ser incluídas tanto no estado quanto em nível regional, para uma melhor representação da diversidade destes organismos em áreas de Caatinga, no semi-árido nordestino.

\section{Agradecimentos}

Ao CNPq pela bolsa de Iniciação Científica (PIBIC) de Aline Anjos Menezes e bolsa de produtividade em pesquisa à Dra. Marcela E. S. Cáceres. À COPES-UFS pela bolsa de IC de Amanda Barreto Xavier Leite e bolsas PICVOL para Amanda Yumi Otsuka e Luciana Santos de Jesus. Ao Dr. Robert Lücking pela confirmação na identificação de algumas espécies.

\section{Referências Bibliográficas}

Archer, A.W. \& Elix, J.A. 2007. New species and new reports in the Australian Graphidaceae. Royal Botanic Gardens and Domain Trust. Telopea 11(4): 451-462.

Awasthi, D.D. 1975. A Monograph of the Lichen Genus Dirinaria. Bibliotheca Lichenologica 2: 1-108.

Benatti, M.N. \& Marcelli, M.P. 2007. Gêneros de fungos liquenizados dos manguezais do sul-sudeste do Brasil, com enfoque no manguezal do Rio Itanhaém, São Paulo. Acta Botanica Brasilica 21: 863-878.

Benatti, M.N. \& Marcelli, M.P. 2008. Canoparmelia sanguinea, a new Parmeliaceae from Brazil. Mycotaxon 106: 435-439.

Büdel, B. \& Scheidegger, C. 2008. Thallus morphology and anatomy. Pp. 40-68. In: Nash, T. III (Ed.) Lichen Biology. 2 ed. Cambridge, Cambridge University Press.

Cáceres, M.E.S. 2007. Corticolous crustose and microfoliose lichens of northeastern Brazil. Libri Botanici 22: 1-168.

Cáceres, M.E.S. \& Lücking, R. 2000. Three new species and one new combination of foliicolous lichens and lichenicolous fungi from the Atlantic Rainforest in Pernambuco state, Brazil. Nova Hedwigia 70(1-2): 217-226.

Cáceres, M.E.S.; Lücking, R. \& Rambold, G. 2007. Phorophyte specificity and environmental parameters versus stochasticity as determinants for species composition of corticolous crustose lichen communities in the Atlantic rainforest of northeastern Brazil. Mycological Progress 6(3):117-136.

Cáceres, M.E.S.; Lücking, R. \& Rambold, G. 2008a. Corticolous Microlichens in Northeastern Brazil: Habitat Differentiation Between Coastal Mata Atlântica, Caatinga and Brejos de Altitude. The Bryologist 111(1): 98-117.

Cáceres, M.E.S.; Lücking, R. \& Rambold, G. 2008b. Efficiency of sampling methods for accurate estimation of species richness of corticolous microlichens in the Atlantic rainforest of northeastern Brazil. Biodiversity and Conservation 17: 1285-1301.

Cáceres, M.E.S.; Maia, L.C. \& Lücking, R. 2000. Foliicolous lichens and their lichenicolous fungi in the atlantic rainforest of Brazil: diversity, ecogeography and conservation. Bibliotheca Lichenologica 75: $47-70$.

Dal-Forno, M. 2009. Família Graphidaceae (Ascomycota Liquenizados) em Restinga em Pontal do Sul, Pontal do Paraná, Paraná. Dissertação de Mestrado. Universidade Federal do Paraná, Curitiba.

Dal-Forno, M. \& Eliasaro, S. 2010a. Four new species of Graphis (Ostropales: Graphidaceae) from Brazil. The Lichenologist 42: 77-81.

Dal-Forno, M. \&Eliasaro, S. 2010b. Two new species of Graphidaceae (lichenized Ascomycota) from Brazil. Mycotaxon 112: 15-20.

Eliasaro, S.; Veiga, P.W. \& Donha, C.G.; Nogueira, L. 2009. Inventário de macroliquens epífitos sobre árvores utilizadas na arborização urbana em Curitiba, Paraná, Brasil: Subsídio para biomonitoramento urbano. Biotemas 22(4): 1-8.
Eliasaro, S. 2008. Parmotrema sanctae-candidae, a new species in the Parmeliaceae (lichenized Ascomycota) from Brazil. Mycotaxon 106: 431-434.

Frisch, A. 2006. The lichen family Thelotremataceae in Africa. A revision with special consideration of the taxa from Cameroon and Tanzania. Bibliotheca Lichenologica 92: 3-370.

GBIF - Global Biodiversity Information Facility. 2010. Version 1.2.6. http://data.gbif.org/html (Acesso em 10/09/2010).

Hawksworth, DL. 1995. Challenges in mycology. Mycological Research 99(1): $127-128$

Jungbluth, P.; Marcelli, M.P. \& Elix, J.A. 2008. Five new species of Bulbothrix (Parmeliaceae) from cerrado vegetation in São Paulo State, Brazil. Mycotaxon 104: 1-13.

Jungbluth, P. \& Marcelli, M.P. 2011a. The Pyxine pungens complex in São Paulo State, Brazil. The Bryologist 114: 166-177.

Jungbluth, P. \& Marcelli, M.P.; Kalb, K. 2011b. A new species and a new record of Pyxine (Physciaceae) with norstictic acid from São Paulo State, Brazil. Mycotaxon 115: 435-442.

Käffer, M.I \& Mazzitelli, S.M.A.M. 2005. Fungos liquenizados corticícolas e terrícolas da área da sub-bacia dos Sinos e Taquari- Antas, RS, Brasil. Acta Botanica Brasilica 19(4): 813-817.

Käffer, M.I.; Cáceres, M.E.S.; Vargas, V.M.F. \& Martins, S.M.A. 2010. Novas ocorrências de liquens corticícolas crostosos para a região sul do Brasil. Acta Botanica Brasilica 24(4): 948-951.

Lemos, A.; Käffer, M.I. \& Martins, S.A. 2007. Composição e diversidade de liquens corticícolas em três diferentes ambientes: Florestal, Urbano e Industrial. Revista Brasileira de Biociências 5(2): 228-230.

Lücking, R. 1998. 'Plasticolous' lichens in a tropical rain forest at La Selva Biological Station, Costa Rica. The Lichenologist 30: 287-301.

Lücking, R. \& Cáceres, M.E.S. 2004. Corticolous species of Trichothelium (Ascomycota: Porinaceae). Mycological Research 108: 571-575.

Lücking, R. \& Rivas-Plata, E. 2008. Clave y Guía Ilustrada Para Géneros de Graphidaceae. Glalia [S.l.] 1: 1-39.

Lücking, R.; Archer, A.W. \& Aptroot, A. 2009. A world-wide key to the genus Graphis (Ostropales: Graphidaceae). The Lichenologist 41(4/5): 1-90.

Lücking, R.; Chaves, J.L.; Sipman, H.J.M.; Umanã, L. \& Aptroot, A. 2008. A first assessment of the Ticolichen biodiversity inventory in Costa Rica: The genus Graphis, with notes on the genus Hemithecium (Ascomycota: Ostropales: Graphidaceae). Fieldiana Botany 46: 1-140.

Marbach, B. 2000. Corticole und lignicole Arten der Flechtengattung Buellia sensu lato in den Subtropen und Tropen. Bibliotheca Lichenologica 74: 1-384.

Marcelli, M.P. 1997. Estudo da diversidade de espécies de fungos liquenizados do Estado de São Paulo. Pp. 1-12. In: Joly, C.A. (Org.). BIOTA São Paulo. São Paulo, Fundação André Toselo.

Marcelli, M.P. \& Canêz, L.S. 2008. Novelties on Southern Brazilian Parmeliaceae. Mycotaxon 105: 225-234.

Marcelli, M.P.; Jungbluth, P. \& Elix, J.A. 2009. Four new species of Punctelia from São Paulo State, Brazil. Mycotaxon 109: 49-61.

Marcelli, M.P.; Canêz, L.S.; Benatti, M.N.; Spielmann, A.A.; Jungbluth, P. \& Elix, J.A. 2011. Taxonomical novelties in Parmeliaceae. Bibliotheca Lichenologica 106: 211-224.

Martins, S.M. de A.; Käffer, M.I. \& Lemos, A. 2008. Liquens como bioindicadores da qualidade do ar numa área de termoelétrica, Rio Grande do Sul, Brasil. Hoehnea 35(3): 425-433.

Mascarenhas, J.C.; Beltrão, B.A. \& Souza Junior, L.C. 2005. Diagnóstico do Município de São José da Tapera. Projeto Cadastro de Fontes de Abastecimento por Água subterrânea, Estado de alagoas. Recife, CPRM/PRODEEM.

Nash, T.H. III. 2008. Introduction. Pp. 1-8. In: Nash, T.H. III (Ed.) Lichen Biology. 2 ed. Cambridge, Cambridge University Press.

Pereira, E. C.; Marcelli, M. P.; Silva, N. H. \& Silva, A. M. 2006. Liquens. Pp. 108-119. In: Porto, K.C.; Tabarelli, M. \& Almeida-Cortez, J. (Org.). Diversidade biológica e conservação da Floresta Atlântica ao norte do rio São Francisco. Recife, Universtitária.

Rivas Plata, E.; Lücking, R.; Aptroot, A.; Sipman, H.J.M.; Chaves, J.L.; Umaña, L. \& Lizano, D. 2006. A first assessment of the Ticolichen biodiversity inventory in Costa Rica: the genus Coenogonium (Ostropales: Coenogoniaceae), with a world-wide key 
and checklist and a phenotypebased cladistic analysis. Fungal Divertsity 23: 255-321.

Spielmann, A.A. 2006. Checklist of Lichens and Lichenicolous Fungi of Rio Grande do Sul (Brazil). Caderno de Pesquisa Série Biologia 18(2): 7-125.

Spielmann, A.A. \& Marcelli, M.P. 2008a. Bulbothrix viatica, a new species of Parmeliaceae from Brazil. Mycotaxon 103: 201-205.
Spielmann, A.A. \& Marcelli, M.P. 2008b. Parmeliaceae (Ascomycota liquenizados) nos barrancos e peraus da encosta da Serra Geral, Vale do rio Pardo, Rio Grande do Sul, Brasil. Iheringia 63(1): 159-169.

Xavier Filho, L.; Cavalcante, W.A. \& Cavalcante, A.A.S. 1983. Estudio del género Coenogonium en Brasil. Boletim da Sociedade Broteriana 56(2): 115-121.

Webster, J. \& Weber, R.W.S. 2007. Introduction to Fungi. $3^{\text {rd }}$. ed. Cambridge, Cambridge University Press. 